\title{
The impact of helical flow on coronary atherosclerotic plaque development
}

\author{
Giuseppe De Nisco $^{\mathrm{a}}$, Ayla Hoogendoorn ${ }^{\mathrm{b}}$, Claudio Chiastra ${ }^{\mathrm{a}}$, Diego Gallo ${ }^{\mathrm{a}}$, Annette M. Kok ${ }^{\mathrm{b}}$, \\ Umberto Morbiducci ${ }^{\mathrm{a}}$, Jolanda J. Wentzel ${ }^{\mathrm{b}, *}$ \\ ${ }^{\text {a }}$ PoliTo ${ }^{B I O}$ Med Lab, Department of Mechanical and Aerospace Engineering, Politecnico di Torino, 10129, Turin, Italy \\ ${ }^{\mathrm{b}}$ Department of Cardiology, Biomedical Engineering, Erasmus MC, 3000, CA, Rotterdam, the Netherlands
}

\section{H I G H L I G H T S}

- In coronaries, blood flow helicity is strongly associated with wall shear stress.

- Exposure to high helicity intensity levels resulted in low wall thickness growth.

- Helical blood flow has a protective role against coronary atherosclerosis.

\section{A R T I C L E I N F O}

\section{Keywords:}

Atherosclerosis

Computational fluid dynamics

Wall shear stress

Plaque progression

\begin{abstract}
A B S T R A C T
Background and aims: Atherosclerosis has been associated with near-wall hemodynamics and wall shear stress (WSS). However, the role of coronary intravascular hemodynamics, in particular of the helical flow (HF) patterns that physiologically develop in those arteries, is rarely considered.

The purpose of this study was to assess how HF affects coronary plaque initiation and progression, definitively demonstrating its atheroprotective nature.

Methods: The three main coronary arteries of five adult hypercholesterolemic mini-pigs on a high fat diet were imaged by computed coronary tomography angiography (CCTA) and intravascular ultrasound (IVUS) at 3 (T1, baseline) and $9.4 \pm 1.9$ (T2) months follow-up. The baseline geometries of imaged coronary arteries $(n=15)$ were reconstructed, and combined with pig-specific boundary conditions (based on in vivo Doppler blood flow measurements) to perform computational fluid dynamic simulations. Local wall thickness (WT) was measured on IVUS images at T1 and T2, and its temporal changes were assessed. Descriptors of HF and WSS nature were computed for each model, and statistically compared to WT data.

Results: HF intensity was strongly positively associated with WSS magnitude $(p<0.001)$. Overall, coronary segments exposed to high baseline levels of HF intensity exhibited a significantly lower WT growth $(p<0.05)$, compared to regions with either mid or low HF intensity.

Conclusions: This study confirms the physiological significance of HF in coronary arteries, revealing its protective role against atherosclerotic WT growth and its potential in predicting regions undergoing WT development. These findings support future in vivo measurement of coronary HF as atherosclerotic risk marker, overcoming current limitations of in vivo WSS assessment.
\end{abstract}

\section{Introduction}

Coronary atherosclerosis is a complex and multifactorial disease, influenced by local biological, biomechanical, and systemic factors $[1,2]$. The mechanisms underlying the role of hemodynamics in promoting the onset and progression of the atherosclerotic disease in coronary arteries are still not completely understood.

Among the biomechanical factors that promote atherosclerotic plaque onset and progression in coronary arteries, local hemodynamics plays a major role [2,3]. In particular, low wall shear stress (WSS) is widely recognized as an independent predictor of plaque development $[4,5]$.

Besides the widely investigated WSS, physiological helical flow (HF) has also been hypothesized to have a relevant impact on vascular disease. HF, consisting of a helical-shaped arrangement of the streaming blood (as given by the combination of translational and rotational blood flow motions), is known to markedly characterize arterial hemodynamics [6-9]. The physiological significance of arterial HF, in

\footnotetext{
* Corresponding author. Molewaterplein 40, 3015 GD Rotterdam, The Netherlands. P.O. Box 2040, 3000, CA, Rotterdam, the Netherlands.

E-mail address: j.wentzel@erasmusmc.nl (J.J. Wentzel).
} 
particular its atheroprotective nature, has emerged in the last decade in the human aorta [6,10-12] and in the human carotid bifurcation [13-15]. All those studies highlighted the role played by HF in mitigating flow disturbances close to the vascular wall, thus protecting from atherosclerosis development by suppressing the amount of low, proatherogenic WSS [2].

Very recently, we showed the existence of distinguishable HF flow features in coronary arteries. These HF features were hypothesized to be atheroprotective, as our data demonstrated a strong association between HF and the luminal surface area exposed to low, proatherogenic WSS [16].

Following these recent findings, the final goal of this study was to demonstrate the protective role of HF for atherosclerotic plaque development over time. Findings from this work would contribute (1) to further clarify the physiological significance of HF in coronary arteries, and (2) to the debate on a possible future use of HF-based hemodynamic descriptors as in vivo surrogate markers of atherogenic WSS for diagnostic/prognostic purposes overcoming current limitations and inaccuracies related to the direct measurement of WSS from in vivo imaging, deriving mainly from poor spatial and temporal resolution [17].

\section{Materials and methods}

\subsection{Animal population and imaging}

Five adult familial hypercholesterolemia Bretoncelles Meishan minipigs with a mutation in the low-density lipoprotein receptor (LDLR) (age of $34 \pm 3$ months, castrated male) were put on a high fat diet to trigger atherosclerosis development. As described in detail elsewhere $[16,18]$, the animals underwent computed coronary tomography angiography (CCTA) and intravascular ultrasound (IVUS) imaging of the three main coronary arteries (left anterior descending - LAD, left circumflex - LCX, and right coronary artery - RCA). The imaging protocol was performed at 3 months after the start of the diet (T1, considered as the baseline in this study), and after $9.4 \pm 1.9$ months (T2). At T1, Doppler-based blood flow velocity measurements were recorded in each artery at the inflow section and immediately upstream and downstream of each side branch, using the ComboWire (Volcano Corp., Rancho Cardova, CA, USA). An overview of the methods is provided in Fig. 1. In addition, some classical risk factors were measured in the 5 investigated animals including weight, leukocytes, total cholesterol, LDL-C, HDL-C and LDL-C/HDL-C ratio.

The study was performed according to the National Institute of Health guide for the Care and Use of Laboratory animals [19]. Ethical approval was obtained from the local animal ethics committee of the Erasmus MC (EMC nr. 109-14-10).

\subsection{Plaque growth measurements}

To quantify the local wall thickness (WT), the lumen and vessel wall contour of each of the 15 investigated coronary arteries (5 LAD, 5 LCX and 5 RCA, Fig. S1 of the Supplementary Materials) were semi-automatically detected on IVUS images at T1 and at T2 using QCU-CMS software (version 4.69, Leiden University Medical Centre, LKEB, Division of Image Processing), as depicted in Fig. 2. WT was assessed by subtracting the distance between the lumen center and the lumen contour, from the distance to the outer wall contour. Plaque development over time was quantified in terms of change in WT $(\Delta \mathrm{WT})$ between time points $\mathrm{T} 1$ and $\mathrm{T} 2$. The $\Delta \mathrm{WT}$ was then adjusted for the number of months between both time points for the individual pig, resulting in a measure of $\Delta \mathrm{WT} /$ month. WT measurements were averaged over $3 \mathrm{~mm} / 45^{\circ}$ sectors of the luminal surface (Fig. 2) in order to capture the local effects of HF on plaque development.

\subsection{Computational hemodynamics}

The 3D geometry of coronary arteries at $\mathrm{T} 1$ was reconstructed by stacking segmented IVUS lumen contours on the CCTA 3D centerline using Mevislab (Bremen, Germany), as described in detail elsewhere [16]. Unsteady-state CFD simulations were performed on the reconstructed geometries to quantify near-wall and intravascular hemodynamic features. The finite volume method was used to numerically solve the governing equations of fluid motion. Blood was assumed as an incompressible, homogeneous, non-Newtonian fluid. No-slip condition was assumed at the arterial wall. Pig tailored boundary conditions were



Fig. 1. Schematic diagram of the study design, showing how imaging data contribute to define vessel geometry and hemodynamic variables. LDRL: low-density lipoprotein receptor; CCTA: coronary computed tomography angiography; IVUS: intravascular ultrasound. 


\section{Hemodynamic descriptors}

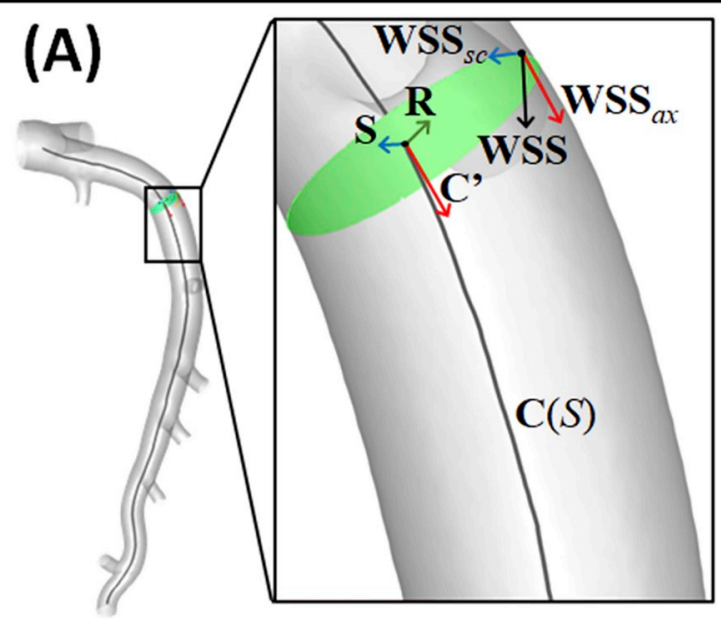

WSS-based descriptors (A)

(B)

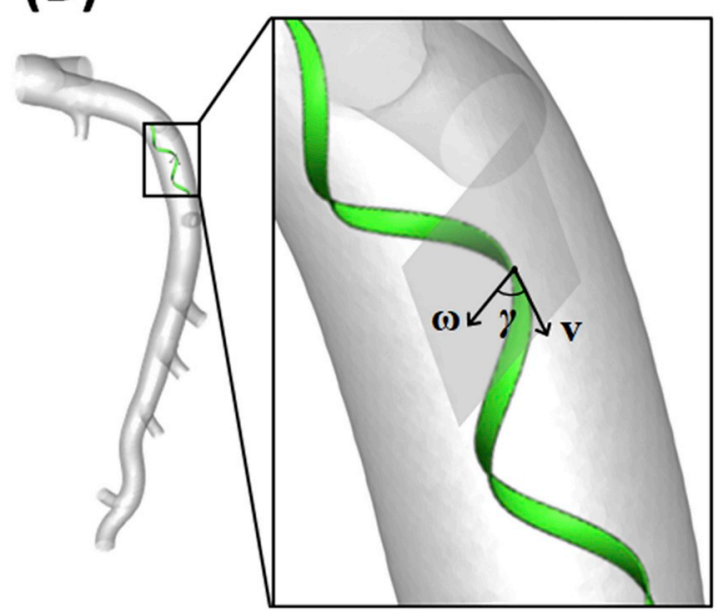

\begin{tabular}{|c|c|}
\hline TAWSS & $\begin{array}{l}\text { Cardiac cycle-average wSS vector (black arrow) } \\
\text { magnitude }\end{array}$ \\
\hline WSS $_{o x}$ & $\begin{array}{l}\text { WSS vector projection along the axial direction (red } \\
\text { arrow). The direction is determined by the direction of } \\
\text { the tangent to the vessel centerline }\left(\mathbf{C}^{\prime}\right)\end{array}$ \\
\hline WSS $_{s c}$ & $\begin{array}{l}\text { WSS vector projection along the secondary direction } \\
\text { (blue arrow). The direction is identified as the } \\
\text { direction perpendicular to the tangent of the vessel } \\
\text { centerline (indicated by S) }\end{array}$ \\
\hline WSS $_{\text {rotio }}$ & $\begin{array}{l}\text { Cardiac cycle-average ratio of } \text { WSS }_{s c} \text { to } \text { WSS }_{a x} \\
\text { magnitudes. If WSS rotio }<1 \text {, the WSS axial projection is } \\
\text { the predominating one }\end{array}$ \\
\hline AvgWSS $_{a x}$ & Cardiac cycle-average WSS $_{a x}$ vector \\
\hline AvgWSS $_{s c}$ & Cardiac cycle-average $\mathbf{W S S}_{\mathbf{s c}}$ vector \\
\hline TAWSS $_{a x}$ & Cardiac cycle-average WSS $_{a x}$ vector magnitude \\
\hline TAWSS $_{s c}$ & Cardiac cycle-average WSS $_{s c}$ vector magnitude \\
\hline
\end{tabular}

HF-based descriptors (B)

A measure of the alignment of local velocity $(\mathbf{v})$ and vorticity $(\omega)$ vectors, given by the cosine of the angle between both vectors $(\gamma)$. By definition, $-1 \leq \mathrm{LNH} \leq 1$,

LNH where: $L N H=-1$ indicates same direction while opposite orientation of $v$ and $\omega$; $L N H=1$ indicates same direction and orientation of $\mathbf{v}$ and $\boldsymbol{\omega}$. LNH sign indicates left- and right-handed helical flow patterns

$h_{2} \quad$ Helicity intensity, i.e., the cardiac cycle-average total amount of helical blood flow intensity

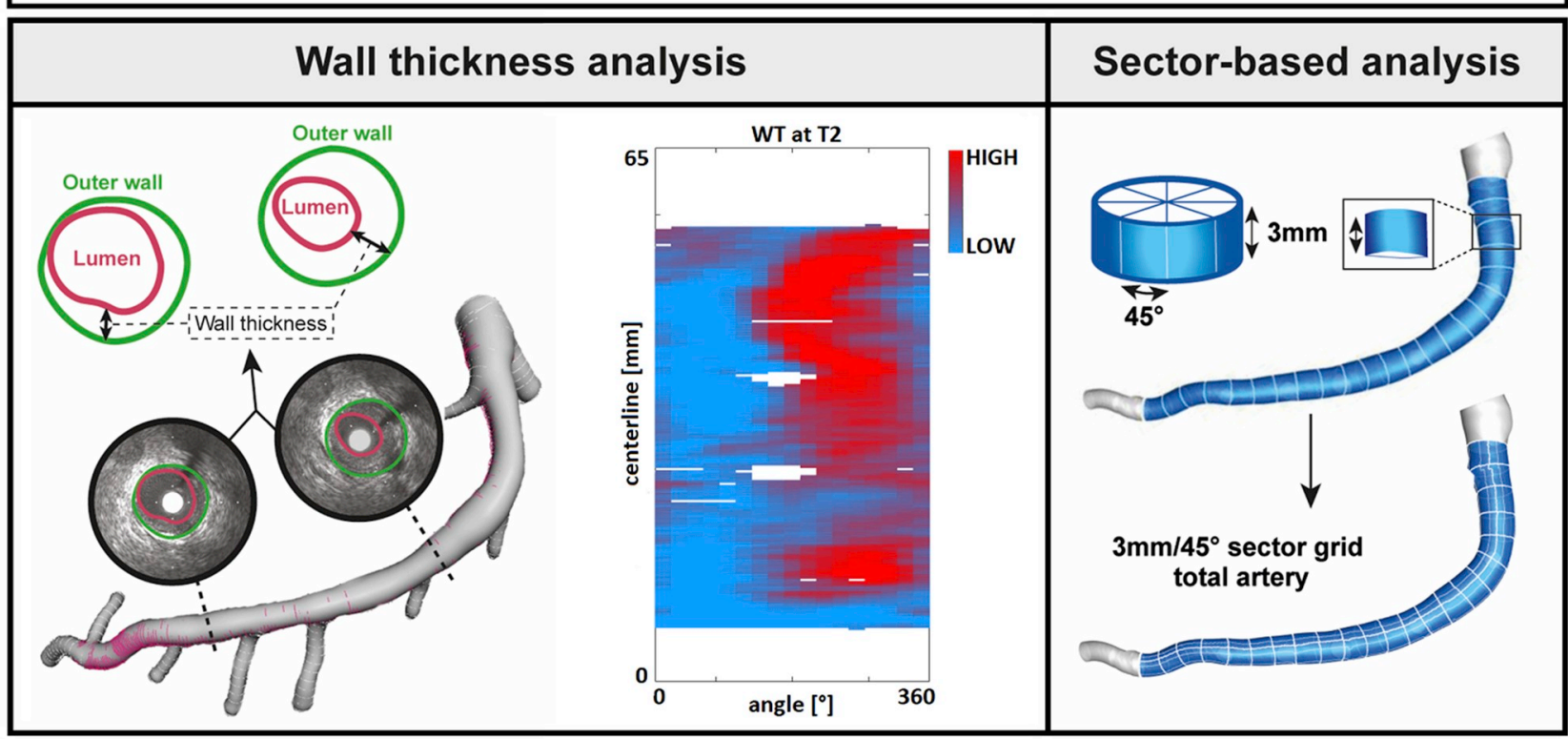

(caption on next page) 
Fig. 2. Methodology of hemodynamic descriptors and wall thickness (WT) assessment and analysis.

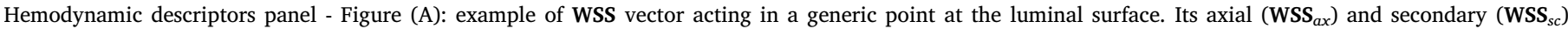

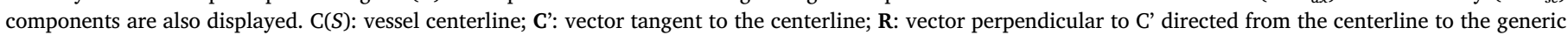

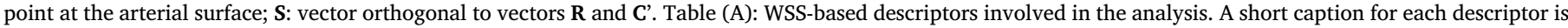

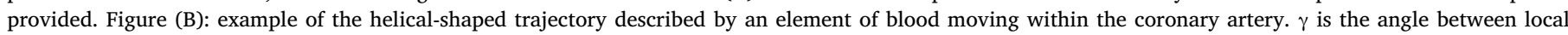

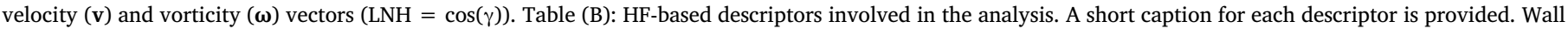



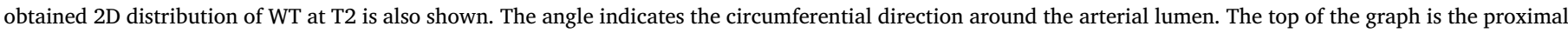

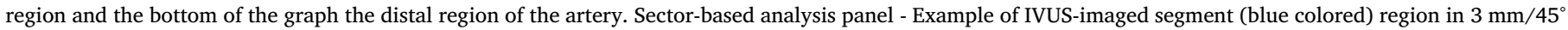

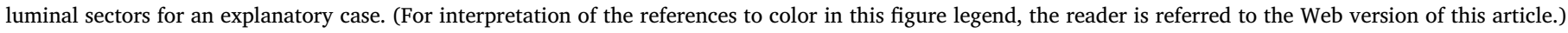

derived from individual in vivo velocity measurements at several locations along the vessel, obtained through a Doppler velocity guidewire (ComboWire; Volcano Corporation). The most proximal measurement was used to estimate the flow rate value, prescribed in the model as inlet boundary condition in terms of time-dependent flat velocity profile. At each side branch, the flow ratio was estimated from the in vivo measurements as the difference between upstream and downstream velocity-based flow rate measurements, and applied as outflow condition. If flow velocity measurements were inaccurate or not available, a diameter-based scaling law [20] was applied to estimate the flow ratio [16].

\subsection{Hemodynamic descriptors}

The hemodynamic descriptors considered for the analysis are listed in Fig. 2 (their mathematical formulation is reported in Table S2 of the Supplementary Materials). In short, HF in the 15 coronary artery models at T1 was assessed in terms of average HF intensity $\left(h_{2}\right)$, which gives a measure of the strength of HF [13]. To characterize the HF intensity in each coronary artery, $h_{2}$ was computed in: (1) the whole volume of the main vessel; (2) a near-wall region, i.e. the intravascular region close to the vessel wall. This near-wall region was defined as the volume of fluid contained in the outer $10 \%$ of the radius of the lumen (see Fig. S2 of the Supplemental data for a visual explanation). The consideration of the near-wall volume was motivated by the idea that HF near the wall might pose a stronger effect on the WSS values than the $\mathrm{HF}$ in the whole intravascular volume.

In addition, the local normalized helicity (LNH) [21] was adopted to visualize right- and left-handed rotating helical flow patterns inside coronary arteries (respectively, positive and negative LNH values, as depicted in Table S2 of the Supplemental data) [11].

To complement the HF characterization, the luminal distribution of time-averaged wall shear stress (TAWSS) and of three descriptors of WSS multidirectionality were evaluated at baseline (Fig. 2 and Table S2). In short, WSS multidirectionality was described considering two projections of WSS vector [22]: (1) along the centerline of the artery, defining the "axial direction" ( WSS $_{a x}$ ); (2) perpendicular to the centerline, defining the secondary direction ( $\mathbf{W S S}_{s c}$ ). The $\mathbf{W S S}_{a x}$ and $\mathbf{W S S}_{s c}$ local vectors were averaged over the cardiac cycle (AvgWSS ${ }_{a x}$ and AvgWSS $_{s c}$, respectively). Moreover, their cycle-average magnitude was evaluated (TAWSS ${ }_{a x}$ and TAWSS sc $_{s c}$, respectively). Additionally, the ratio of the magnitudes of secondary over the axial WSS components $\left(\mathrm{WSS}_{\text {ratio }}\right.$ ) was computed (Fig. 2 and Table S2) [22]. This latter descriptor is useful to determine whether WSS is predominantly aligned with the vessel centerline (WSS $a x$ ) or with the direction perpendicular to it $\left(\mathbf{W S S}_{s c}\right.$ ).

For the analysis, to capture the local effect of the WSS- and HFbased descriptors on WT, the data were averaged over $3 \mathrm{~mm} / 45^{\circ}$ sectors (see the sector-based analysis panel in Fig. 2). For the HF-based descriptors, this included both the near-wall (outer 10\%) and entire volumes of the sectors.

\subsection{Statistical analysis}

The existence of possible associations between WSS and HF was investigated considering the average values of the WSS-based descriptors and the HF-based descriptor $h_{2}$ over each individual coronary artery. Regression analysis was used to identify relations between each pair of hemodynamic descriptors and reported as Spearman correlation coefficients.

The analysis of the relation between plaque growth and hemodynamic descriptors was conducted using the sector-based data applying a mixed model with hemodynamic descriptors as fixed factors, the individual vessel as random factor to correct for clustering of the analyzed sectors per vessel and the average cholesterol levels as covariate (IBM SPSS Statistics, version 24.0). The values of the hemodynamic descriptors were classified as low, mid or high, based on artery-specific tertile-division. Statistical significance was assumed for $p<0.05$.

\section{Results}

Classical cardiovascular risk factors, weight, leukocytes, cholesterol, LDL-C, HDL-C and LDL-C/HDL-C ratio did not significantly change over time for the investigated 5 pigs and are presented in Table S1 of the Supplementary Materials.

\subsection{Coronary hemodynamics: general observations}

For each investigated coronary artery model, the distribution of the WSS-based descriptors was assessed, as shown for a representative case in Fig. 3A-D. A similar approach was used for studying the HF features: the LNH red and blue colors indicate right-handed and left-handed HF patterns, respectively. Thereby, the presence of two distinguishable counter-rotating HF patterns was observed in this case (Fig. 3E) and reflects the arrangement in counter-rotating helical structures in all coronary arteries.

Fig. 3A shows the luminal distribution of TAWSS highlighting, as expected, the presence of case-specific focal low TAWSS regions located at the distal portion of the main branch. Furthermore, the figure shows in the other panels (B and C) that WSS was predominantly aligned with the forward flow direction (i.e., positive AvgWSS ${ }_{a x}$ values), which was representative for all cases. Moreover, it emerged that the organization of coronary blood flow in two counter-rotating helical structures, which is evident from LNH visualization, influences the near-wall hemodynamics of coronary vessels. Considering the coronary artery depicted in Fig. 3D, positive/negative values of AvgWSS sc, indicating right-handed and left-handed directions respectively, resemble the rotating direction of helical flow structures given by the LNH (Fig. 3E). In addition, the

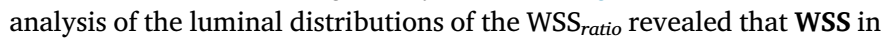
the axial direction ( WSS $_{a x}$ ) was dominant over the WSS perpendicular to the vessel centerline $\left(\mathbf{W S S}_{s c}\right.$ ). In fact, the WSS $_{\text {ratio }}$ was $<1$ over most of the lumen of all the investigated coronary arteries (around $94 \%$ of the investigated luminal surface sectors, see also Fig. 3B).

\subsection{Link between hemodynamic variables}

To assess whether HF could represent a reliable surrogate marker for WSS descriptors, we analyzed the association between HF-based $v s$. WSS-based descriptors. The analysis revealed a significant association between the mean values of the WSS-based descriptors and helicity 
(A)



(B)

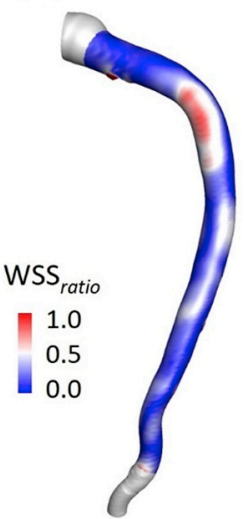

(C)

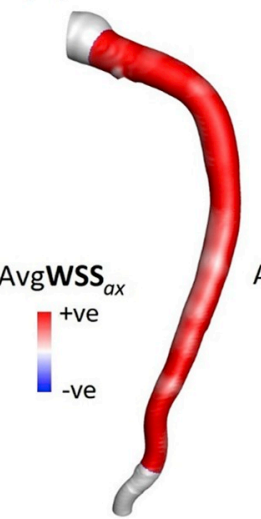

(D)

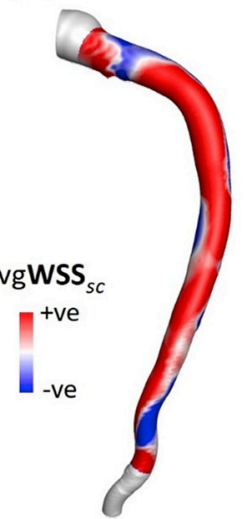

(E)

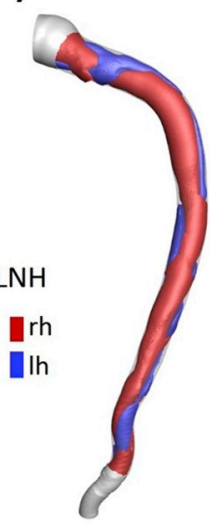

(F)



Fig. 3. Coronary hemodynamics: general observations and link between hemodynamic variables.

(A-D) WSS-based descriptors distribution at the luminal surface of a representative LAD coronary artery (a) (see Fig. S1 of the Supplementary Materials). For the same explanatory case, visualization of LNH cycleaverage isosurfaces is also provided in panel (E). For TAWSS (WSS ${ }_{\text {ratio }}$ ), the low and high values are dispayed by red (blue) and blue (red), respectively. For cycle-average axial WSS vector projections (AvgWSS ${ }_{a x}$ ), colors identify the forward (red) and backward (blue) flow direction, respectively. As for $\mathrm{LNH}$, also for the cycle-average secondary WSS vector projections (AvgWSS ${ }_{s c}$ ) blue and red colors identify the left and righthanded direction, respectively. + ve: positive; -ve: negative; rh: right-handed; lh: lefthanded. (F) Spearman correlation coefficients between WSS-based and HF-based descriptors. The average value of the hemodynamic descriptors for each individual case was considered. For statistically significant relations, $p$ values are also reported. (For interpretation of the references to color in this figure legend, the reader is referred to the Web version of this article.) intensity $h_{2}$ (Fig. 3F). In detail, TAWSS was strongly and positively associated with both whole volume $h_{2}(\mathrm{r}=0.925, p<0.001)$ and near-wall $h_{2}(\mathrm{r}=0.629, p<0.01)$. This indicates that higher values of helicity intensity $\left(h_{2}\right)$ correspond to higher TAWSS values. Moreover, positive associations emerged between the whole volume $h_{2}$, and both TAWSS $_{a x}(\mathrm{r}=0.843, p<0.001)$ and TAWSS $s c(\mathrm{r}=0.843$, $p<0.001$ ), supporting the idea that high $h_{2}$ values in the whole intravascular lumen induce higher TAWSS values, both along axial direction and perpendicular to it (secondary direction).

A weaker, but still significant, positive association emerged between near-wall $h_{2}$ and TAWSS ${ }_{a x}(\mathrm{r}=0.629, p<0.05)$. For the TAWSS $s c$, only a near-significant association was observed with the near-wall $h_{2}$ ( $r=0.468, p=0.081)$. This result suggests a less important role for near-wall $h_{2}$, compared to whole volume $h_{2}$, in determining secondary WSS magnitude. This is confirmed by the direct, significant association between the whole volume $h_{2}$ and WSS $_{\text {ratio }}(\mathrm{r}=0.757, p<0.01$ ), while no significant association was observed for the latter with nearwall $h_{2}$. Therefore, $h_{2}$ in the whole intravascular volume seems to influence the relative weight of the magnitude of secondary $v s$. axial WSS components.

\subsection{Link between hemodynamic variables and increase in wall thickness}

Overall, the 15 pig coronary arteries presented a marked increase in average WT over the follow-up period (WT at $\mathrm{T} 1=0.183 \pm 0.108 \mathrm{~mm}, \mathrm{WT}$ at $\mathrm{T} 2=0.427 \pm 0.313 \mathrm{~mm}$; $p<0.001$ ).

Coronary sectors exposed to low TAWSS exhibited a significantly larger $\Delta$ WT per month $(0.048 \pm 0.007 \mathrm{~mm} / \mathrm{month})$ compared to regions with either mid $(\Delta \mathrm{WT} /$ month $=0.035 \pm 0.007 \mathrm{~mm} / \mathrm{month})$ or high $(\Delta \mathrm{WT} /$ month $=0.027 \pm 0.007 \mathrm{~mm} / \mathrm{month})$ TAWSS values (Fig. 4, top panel). The analysis revealed a significant, inverse association between HF and WT progression. In particular (Fig. 4, top panel), in luminal sectors where near-wall $h_{2}$ was high, significantly low WT growth rate $(0.032 \pm 0.007 \mathrm{~mm} / \mathrm{month})$ was observed, compared to luminal sectors with either mid $(\Delta \mathrm{WT} / \mathrm{month}=0.037 \pm$ $0.007 \mathrm{~mm} / \mathrm{month})$ or low $(\Delta \mathrm{WT} / \mathrm{month}=0.040 \pm 0.007 \mathrm{~mm} /$ month) near-wall $h_{2}$. A similar relation emerged for $h_{2}$. Among the investigated descriptors of WSS directionality, only high TAWSS $a x$ was significantly associated with lower WT progression $\left(0.030 \pm 0.002 \mathrm{~mm} /\right.$ month for the highest TAWSS ${ }_{a x}$ tertile).

In addition, the results of the time-specific statistical analysis are reported in Fig. 4 (T1 - mid panel; T2, bottom panel). In detail, the association between $h_{2}$ and WT at T1 was only near significant ( $p=0.06$ ), while no significant association emerged between near-wall $h_{2}$ and measured WT at T1 (Fig. 4, mid panel). As for WSS distribution, luminal sectors exposed to high TAWSS at T1 significantly displayed the lowest T1 WT values. Similar results (but with smaller standard errors), were observed for TAWSS $a x$. However, neither TAWSS $s$ nor WSS were significantly associated with WT at T1. The analysis of the relations between hemodynamic descriptors at $\mathrm{T} 1$ and $\mathrm{WT}$ at $\mathrm{T} 2$ revealed similar results to those found for the overall WT growth per month between $\mathrm{T} 1$ and $\mathrm{T} 2$ (Fig. 4, lower panel). In contrast to the $\Delta \mathrm{WT} / \mathrm{month}$ analysis, in the analysis of WT at T2, luminal sectors exposed to higher TAWSS $_{s c}$ values at T1 exhibited significantly lower WT values at T2.

\section{Discussion}

\subsection{Summary of findings and their implications}

In the present study, we investigated the association between local hemodynamics and atherosclerosis progression in a representative dataset of 15 pig coronary arteries. The study highlighted the existence of a clear association between HF intensity $\left(h_{2}\right)$ at baseline and plaque development over time in coronary arteries. In detail, sectors at the luminal surface with the lowest WT growth rate values were preceded by higher baseline values of HF intensity, suggesting a beneficial role of the HF patterns in coronary arteries. The atheroprotective role of HF was confirmed when extending the analysis to WSS, a factor known to be involved in atherosclerotic disease [23]. These findings confirm and strengthen our previously reported associations between HF intensity and WSS-based hemodynamic descriptors in coronary arteries [16].

A schematic of the main findings is reported in Fig. 5, putting HF in the cascade of events determining atherosclerotic plaque development 



Fig. 4. Link between hemodynamic variables and increase in wall thickness.

Relationship between baseline (T1) hemodynamic descriptor levels and 1) estimated plaque growth per month (top panel), 2) WT at T1 (middle panel), and 3) WT at T2 (bottom panel). Estimated mean and standard error of the mean (SEM) values are reported. The hemodynamic descriptors were divided in low (dark blue bars), mid (blue bars) and high (light blue bars) tertiles per artery. The average value of the hemodynamic descriptors and WT measurements in the $3 \mathrm{~mm} / 45^{\circ}$ sectors was considered. ${ }^{*} p<0.05$ compared to low tertile, ${ }^{*} p<0.05$ compared to the mid tertile of all parameters. (For interpretation of the references to color in this figure legend, the reader is referred to the Web version of this article.)

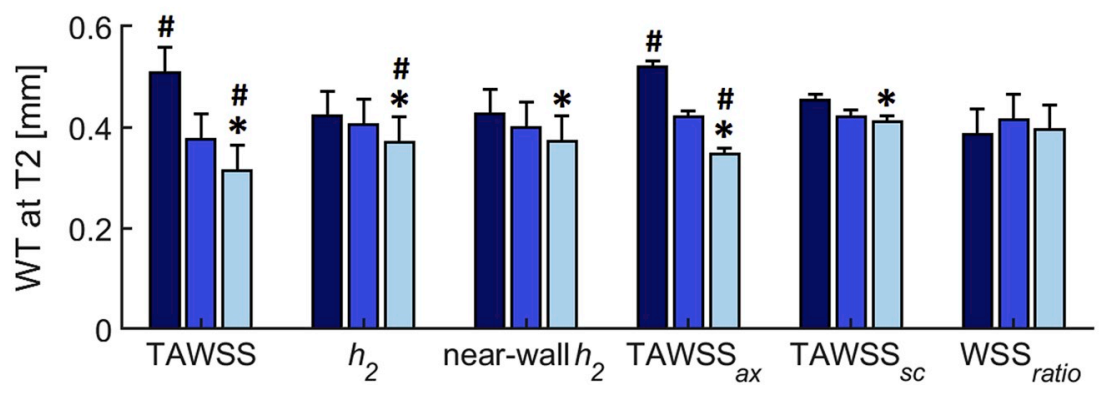

in coronary arteries. Summarizing the suggested hemodynamics-related mechanism involved in atherosclerotic disease progression: (1) helical blood flow patterns characterized by high HF intensity $\left(h_{2}\right)$ stabilize coronary hemodynamics, thus reducing flow disturbances resulting in more atheroprotective high WSS levels at the luminal surface (e.g., Fig. 3-F); (2) atheroprotective WSS values maintain endothelial cells (EC) quiescence and junctions stability [3,23,24], contributing to prevent plaque initiation. The present findings suggest that $\mathrm{HF}$ has a causative role in determining WSS patterns at the luminal surface. Moreover, the already highlighted role of low WSS as predictor of plaque development in human [5] and animal [18,25] studies clearly emerged also in this study (Fig. 4 - top panel). In detail, low baseline values of TAWSS, which might be determined by low HF intensity (Fig. 3-F), potentially trigger biological mechanisms like EC polygonal shaping, pro-atherogenic genes upregulation, nitric oxide reduction. These biological mechanisms induce EC dysfunction [3,23,24,26] and promote the atherogenic plaque onset and faster disease progression.

In this study, the commonly used multidirectional WSS metric oscillatory shear index (OSI) was not analyzed since previous studies demonstrated that coronary arteries develop very low OSI values $[16,18]$. Moreover, the already observed scarce multidirectionality of WSS in ostensibly healthy (time point T1) coronary vessels [16] was confirmed here by assessing its axial and secondary components. The WSS $_{\text {ratio }}$ adopted values lower than 1 over most of the lumen of all the investigated coronary arteries, indicating that the WSS is markedly aligned with the main flow direction (see explanatory case in Fig. 3-B). This finding suggests a primary role of low over multidirectional WSS in promoting the early stage of atherosclerotic disease, consistently with previous observations [18]. A significant role of multidirectional WSS in plaque development is expected to emerge in later stages of the disease $[15,27,28]$.

The association of hemodynamic quantities with WT at T1 was significant only for TAWSS and TAWSS ${ }_{a x}$. Even though plaque growth was just initiated, the plaque location showed to comply with the local TAWSS. Luminal regions exposed to higher TAWSS $s c$ values were significantly associated with lower WT at T2, reflecting that high (atheroprotective) values of TAWSS generally result in higher values of TAWSS $_{s c}$.

Furthermore, the results of this study serve to quantitatively explain for the first time the irregular helical-shaped distribution of fatty and fibrous plaques in coronary artery reported by previous ex vivo studies [29-32], and hinted at by the WT patterns shown for the representative case in Fig. 2, where the high WT region seems to follow a helical distribution. The present findings may support the hypothesis that the helical patterns of plaque occurrence are explained by the helical patterns of WSS (Fig. 3), which are a consequence of the HF patterns in the intravascular volume.

\subsection{Limitations of the study}

Several limitations could weaken the findings of this study. Computational hemodynamic modelling suffers from assumptions and uncertainties, such us rigid walls and absence of the cardiac-induced motion of coronary arteries. However, their impact on the WSS distribution has been demonstrated to be minor, especially when considering time-averaged WSS quantities [33]. Moreover, the findings of the study are based upon a relatively modest number of coronary artery models $(\mathrm{N}=15)$. Nevertheless, the consideration of multiple sectors 




Fig. 5. Schematic of the main findings of the study and the relationship with biological mechanisms related to atherosclerosis.

The "Hemodynamics" panel reports the relations among the investigated hemodynamic descriptors; the "Biological mechanisms" panel reports the well-known biological effects caused by atheroprotective or atheroprone hemodynamics (colored in yellow and red, respectively). The black arrows, indicating the cause-effect relation between HF- and WSS-based hemodynamic descriptors, emphasize that HF "shapes" WSS, which, in turn, interacts with the pathophysiology of atherosclerotic plaque formation. (For interpretation of the references to color in this figure legend, the reader is referred to the Web version of this article.)

within each coronary artery allowed for statistically significant relationships to emerge, capturing the links between local hemodynamics, WT, and WT progression, when using a linear mixed-effects model correcting for intra-vessel and cholesterol WT dependence. The here adopted division of the hemodynamic variables in tertiles could be considered arbitrary. However, the lack of established threshold values justifies this objective and conservative choice. Lastly, this study was carried out on a pig model. However, the established similarity between pig and human coronary anatomy and hemodynamics [34] supports the translation of the findings of this study to human coronary arteries.

\subsection{Future perspectives}

In addition to the causative role of helical flow in determining WSS, a beneficial relation between HF intensity at baseline and WT and its progression in the follow-up emerged here. Taken together, these findings suggest that HF intensity may serve as a convenient and pragmatic surrogate marker of low WSS for prediction of WT progression. Although WSS remains the more sensitive hemodynamic indicator for atherosclerotic disease, in vivo WSS measurements could be less accurate than measurements of intravascular fluid quantities like HF, as a consequence of a higher sensitivity to noise, lumen edge definition, spatial and temporal resolution [35,36]. Given the causative role of $\mathrm{HF}$ in determining WSS [16], we here suggest the use of HF intensity as surrogate marker of plaque onset and progression in coronary arteries. Future advances in phase-contrast magnetic resonance imaging are expected to extend the feasibility of in vivo arterial helical flow quantitative analysis, already demonstrated for large arteries [6,11,37-39], to small vessels like the coronary arteries $[17,40-44]$. This would allow non-invasive in vivo-based prediction of atherosclerotic disease progression based upon HF-based descriptors and thereby open a clinical translation of the relationships reported in this study.

\subsection{Conclusions}

This study on coronary arteries confirms a clear association between helical flow, anti-atherogenic wall shear stress patterns and protection from plaque progression over time in an atherosclerotic pig model. In detail, the study confirms the role of helical blood flow features (in terms of HF intensity) in conditioning WSS luminal distribution, which in turn interacts with the pathophysiology of atherosclerotic plaque formation. Therefore, in the cascade of hemodynamic events related to the atherosclerotic disease, intravascular HF influences the WSS patterns at the vascular wall, and, hence, the spatial distribution of the atherosclerotic plaque. Due to the causative role of $\mathrm{HF}$ in determining WSS luminal distribution, low HF intensity could act as a practical surrogate marker of low WSS and, thus, as a potential biomechanical predictor of atherosclerotic plaque onset and progression.

\section{Financial support}

Funding was received from the European Research Council under the European Union's Seventh Framework Programme/ERC Grant Agreement n. 310457.

\section{Author contributions}

G.D.N., A.H., C.C, D.G., U.M. and J.J.W.: conception and design of the study; A.H.: acquisition and analysis of in vivo data; G.D.N. and A.M.K.: computational simulation and analysis of simulation data; G.D.N, D.G., C.C., U.M., and J.J.W.: drafting of the manuscript. All authors revised the manuscript critically for important intellectual content and provided final approval for publication.

\section{Declaration of competing interest}

The authors state no conflict of interest for the study object of the manuscript. The research was not supported financially by private companies. None of the authors has a financial agreement with peoples or organizations that could inappropriately influence their work.

\section{Appendix A. Supplementary data}

Supplementary data to this article can be found online at https:// doi.org/10.1016/j.atherosclerosis.2020.01.027.

\section{References}

[1] B.R. Kwak, M. Bäck, M.L. Bochaton-Piallat, G. Caligiuri, M.J. Daemen, P.F. Davies, I.E. Hoefer, P. Holvoet, H. Jo, R. Krams, S. Lehoux, C. Monaco, S. Steffens, R. Virmani, C. Weber, J.J. Wentzel, P.C. Evans, Biomechanical factors in atherosclerosis: mechanisms and clinical implications, Eur. Heart J. 35 (43) (2014) 3013-3020.

[2] U. Morbiducci, A.M. Kok, B.R. Kwak, P.H. Stone, D.A. Steinman, J.J. Wentzel, Atherosclerosis at arterial bifurcations: evidence for the role of haemodynamics and geometry, Thromb. Haemostasis 115 (3) (2016) 484-492.

[3] J.J. Wentzel, Y.S. Chatzizisis, F.J.H. Gijsen, G.D. Giannoglou, C.L. Feldman, P.H. Stone, Endothelial shear stress in the evolution of coronary atherosclerotic 
plaque and vascular remodelling: current understanding and remaining questions, Cardiovasc. Res. 96 (2012) 234-243.

[4] K.C. Koskinas, G.K. Sukhova, A.B. Baker, M.I. Papafaklis, Y.S. Chatzizisis, A.U. Coskun, T. Quillard, M. Jonas, C. Maynard, A.P. Antoniadis, G.P. Shi, P. Libby, E.R. Edelman, C.L. Feldman, P.H. Stone, Thin-capped atheromata with reduced collagen content in pigs develop in coronary arterial regions exposed to persistently low endothelial shear stress, Arterioscler. Thromb. Vasc. Biol. 33 (7) (2013) 1494-1504.

[5] P.H. Stone, S. Saito, S. Takahashi, Y. Makita, S. Nakamura, T. Kawasaki, A. Takahashi, T. Katsuki, S. Nakamura, A. Namiki, A. Hirohata, T. Matsumura, S. Yamazaki, H. Yokoi, S. Tanaka, S. Otsuji, F. Yoshimachi, J. Honye, D. Harwood, M. Reitman, A.U. Coskun, M.I. Papafaklis, C.L. Feldman, PREDICTION investigators. Prediction of progression of coronary artery disease and clinical outcomes using vascular profiling of endothelial shear stress and arterial plaque characteristics. The PREDICTION study, Circulation 126 (2012) 172-181.

[6] U. Morbiducci, R. Ponzini, G. Rizzo, M. Cadioli, A. Esposito, F.M. Montevecchi, A. Redaelli, Mechanistic insight into the physiological relevance of helical blood flow in the human aorta. An in vivo study, Biomech. Model. Mechanobiol. 10 (2011) 339-355.

[7] X. Liu, A. Sun, Y. Fan, X. Deng, Physiological significance of helical flow in the arterial system and its potential clinical applications, Ann. Biomed. Eng. 43 (1) (2015) 3-15.

[8] P.A. Stonebridge, P.R. Hoskins, P.L. Allan, J.F. Belch, Spiral laminar flow in vivo, Clin. Sci. 91 (1) (1996) 17-21.

[9] P.A. Stonebridge, S.A. Suttie, R. Ross, J. Dick, Spiral laminar flow: a survey of a three-dimensional arterial flow pattern in a group of volunteers, Eur. J. Vasc. Endovasc. Surg. 52 (5) (2016) 674-680.

[10] X. Liu, F. Pu, Y. Fan, X. Deng, D. Li, S. Li, A numerical study on the flow of blood and the transport of LDL in the human aorta: the physiological significance of the helical flow in the aortic arch, Am. J. Physiol. Heart Circ. Physiol. 297 (2009) H163-H170.

[11] U. Morbiducci, R. Ponzini, G. Rizzo, M. Cadioli, A. Esposito, F. De Cobelli, A. Del Maschio, F.M. Montevecchi, A. Redaelli, In vivo quantification of helical blood flow in human aorta by time-resolved three-dimensional cine phase contrast MRI, Ann. Biomed. Eng. 37 (2009) 516-531.

[12] U. Morbiducci, R. Ponzini, D. Gallo, C. Bignardi, G. Rizzo, Inflow boundary conditions for image-based computational hemodynamics: impact of idealized versus measured velocity profiles in the human aorta, J. Biomech. 46 (2013) 102-109.

[13] D. Gallo, D.A. Steinman, P.B. Bijari, U. Morbiducci, Helical flow in carotid bifurcation as surrogate marker of exposure to disturbed shear, J. Biomech. 45 (2012) 2398-2404.

[14] D. Gallo, D.A. Steinman, U. Morbiducci, An insight into the mechanistic role of the common carotid artery on the hemodynamics at the carotid bifurcation, Ann. Biomed. Eng. 43 (2015) 68.

[15] D. Gallo, P.B. Bijari, U. Morbiducci, Y. Qiao, Y. Xie, M. Etesami, D. Haabets, E.G. Lakatta, B.A. Wasserman, D.A. Steinman, Segment-specific associations between local haemodynamic and imaging markers of early atherosclerosis at the carotid artery: an in vivo human study, J. R. Soc. Interface 15 (2018) 20180352.

[16] G. De Nisco, A.M. Kok, C. Chiastra, D. Gallo, A. Hoogendoorn, F. Migliavacca, J.J. Wentzel, U. Morbiducci, The atheroprotective nature of helical flow in coronary arteries, Ann. Biomed. Eng. 47 (2) (2019) 425-438.

[17] M. Markl, S. Schnell, C. Wu, E. Bollache, K. Jarvis, A.J. Barker, J.D. Robinson, C.K. Rigsby, Advanced flow MRI: emerging techniques and applications, Clin. Radiol. 71 (8) (2016) 779-795.

[18] A. Hoogendoorn, A.M. Kok, E.M.J. Hartman, G. De Nisco, L. Casadonte, C. Chiastra, A. Coenen, S.A. Korteland, K. Van der Heiden, F.J.H. Gijsen, D.J. Duncker, A.F.W. van der Steen, J.J. Wentzel, Multidirectional wall shear stress promotes advanced coronary plaque development - comparing five shear stress metrics, Cardiovasc. Res. (2019), https://doi.org/10.1093/cvr/cvz212 cvz212.

[19] National Research Council (US), Committee for the Update of the Guide for Care and Use of Laboratory Animals, Guide for the Care and Use of Laboratory Animals, eighth ed., National Academies Press (US), Washington, DC, 2011.

[20] Y. Huo, G.S. Kassab, Intraspecific scaling laws of vascular trees, J. R. Soc. Interface 9 (66) (2012) 190-200.

[21] U. Morbiducci, R. Ponzini, M. Grigioni, A. Redaelli, Helical flow as fluid dynamic signature for atherogenesis in aortocoronary bypass. A numeric study, J. Biomech. 40 (2007) 519-534.

[22] U. Morbiducci, D. Gallo, S. Cristofanelli, R. Ponzini, M.A. Deriu, G. Rizzo, D.A. Steinman, A rational approach to defining principal axes of multidirectional wall shear stress in realistic vascular geometries, with application to the study of the influence of helical flow on wall shear stress directionality in aorta, J. Biomech. 48 (6) (2015) 899-906.

[23] A.M. Malek, S.L. Alper, I. Izumo, Hemodynamic shear stress and its role in atherosclerosis, J. Am. Med. Assoc. 282 (1999) 2035-2042.

[24] Y.S. Chatzizisis, A.U. Coskun, M. Jonas, E.R. Edelman, C.L. Feldman, P.H. Stone,
Role of endothelial shear stress in the natural history of coronary atherosclerosis and vascular remodeling, J. Am. Coll. Cardiol. 49 (2007) 2379-2393.

[25] C. Cheng, D. Tempel, R. van Haperen, A. van der Baan, F. Grosveld, M.J. Daemen, R. Krams, R. de Crom, Atherosclerotic lesion size and vulnerability are determined by patterns of fluid shear stress, Circulation 113 (23) (2006) 2744-2753.

[26] Y.C. Chen, A.V. Bui, J. Diesch, R. Manasseh, C. Hausding, J. Rivera, I. Haviv, A. Agrotis, N.M. Htun, J. Jowett, C.E. Hagemeyer, R.D. Hannan, A. Bobik, K. Peter, A novel mouse model of atherosclerotic plaque instability for drug testing and mechanistic/therapeutic discoveries using gene and microRNA expression profiling, Circ. Res. 113 (3) (2013) 252-265.

[27] H.A. Himburg, D.M. Grzybowski, A.L. Hazel, J.A. LaMack, X.M. Li, M.H. Friedman, Spatial comparison between wall shear stress measures and porcine arterial endothelial permeability, Am. J. Physiol. Heart Circ. Physiol. 286 (5) (2004) H1916-H1922.

[28] A.M. Kok, D.S. Molony, L.H. Timmins, Y.A. Ko, E. Boersma, P. Eshtehardi, J.J. Wentzel, H. Samady, The influence of multidirectional shear stress on plaque progression and composition changes in human coronary arteries, EuroIntervention 15 (8) (2019) 692-699.

[29] B. Fox, K. James, B. Morgan, W.A. Seed, Distribution of fatty and fibrous plaques in young human coronary arteries, Atherosclerosis 41 (1982) 337-347.

[30] T. Nakashima, Y. Iwanaga, Y. Nakaura, Pathologic study of hypertensive heart, Acta Pathol. Jpn. 14 (1) (1964) 129-141.

[31] T. Nakashima, T. Tashiro, Early morphologic stage of human coronary atherosclerosis, Kurume Med. J. 15 (4) (1968) 235-242.

[32] H.N. Sabbah, F.J. Walburn, P.D. Stein, Patterns of flow in the left coronary artery, J. Biomech. Eng. 106 (3) (1984) 272-279.

[33] R. Torii, J. Keegan, N.B. Wood, A.W. Dowsey, A.D. hughes, G.Z. Yang, D.N. Firmin, S.A. Thom, X.Y. Xu, MR image-based geometric and hemodynamic investigation of the right coronary artery with dynamic vessel motion, Ann. Biomed. Eng. 38 (2010) 2606-2620.

[34] L.C. Winkel, A. Hoogendoorn, R. Xing, J.J. Wentzel, K. Van der Heiden, Animal models of surgically manipulated flow velocities to study shear stress-induced atherosclerosis, Atherosclerosis 241 (2015) 100-110.

[35] A. Frydrychowicz, A. Berger, A. Munoz Del Rio, M.F. Russe, J. Bock, A. Harloff, M. Markl, Interdependencies of aortic arch secondary flow patterns, geometry, and age analysed by 4-dimensional phase contrast magnetic resonance imaging at 3 Tesla, Eur. Radiol. 22 (5) (2012) 1122-1130.

[36] U. Morbiducci, R. Ponzini, G. Rizzo, M.E. Biancolini, F. Iannaccone, D. Gallo, A. Redaelli, Synthetic dataset generation for the analysis and the evaluation of image-based hemodynamics of the human aorta, Med. Biol. Eng. Comput. 50 (2) (2012) 145-154.

[37] A. Harloff, F. Albrecht, J. Spreer, A.F. Stalder, J. Bock, A. Frydrychowicz, J. Schöllhorn, A. Hetzel, M. Schumacher, J. Hennig, M. Markl, 3D blood flow characteristics in the carotid artery bifurcation assessed by flow-sensitive 4D MRI at 3T, Magn. Reson. Med. 61 (1) (2009) 65-74.

[38] M. Schäfer, A.J. Barker, V. Kheyfets, K.R. Stenmark, J. Crapo, M.E. Yeager, U. Truong, J.K. Buckner, B.E. Fenster, K.S. Hunter, Helicity and vorticity of pulmonary arterial flow in patients with pulmonary hypertension: quantitative analysis of flow formations, J. Am. Heart Assoc. 6 (12) (2017) e007010.

[39] T.H. Oechtering, M.M. Sieren, P. Hunold, A. Hennemuth, M. Huellebrand, M. Scharfschwerdt, D. Richardt, H.H. Sievers, J. Barkhausen, A. Frydrychowicz, Time-resolved 3-dimensional magnetic resonance phase contrast imaging (4D Flow MRI) reveals altered blood flow patterns in the ascending aorta of patients with valve-sparing aortic root replacement, J. Thorac. Cardiovasc. Surg. S0022-5223 (19) (2019) 30773-1.

[40] A. Brandts, S.D. Roes, J. Doornbos, R.G. Weiss, A. de Roos, M. Stuber, J.J. Westenberg, Right coronary artery flow velocity and volume assessment with spiral k-space sampled breathhold velocity-encoded MRI at 3 tesla: accuracy and reproducibility, J. Magn. Reson. Imag. 31 (5) (2010) 1215-1223.

[41] J. Keegan, P.D. Gatehouse, R.H. Mohiaddin, G.Z. Yang, D.N. Firmin, Comparison of spiral and FLASH phase velocity mapping, with and without breath-holding, for the assessment of left and right coronary artery blood flow velocity, J. Magn. Reson. Imag. 19 (1) (2004) 40-49.

[42] K. Johnson, P. Sharma, J. Oshinski, Coronary artery flow measurement using navigator echo gated phase contrast magnetic resonance velocity mapping at $3.0 \mathrm{~T}, \mathrm{~J}$. Biomech. 41 (3) (2008) 595-602.

[43] C. Jahnke, R. Manka, S. Kozerke, S. Kozerke, B. Schnackenburg, R. Gebker, N. Marx, I. Paetsch, Cardiovascular magnetic resonance profiling of coronary atherosclerosis: vessel wall remodelling and related myocardial blood flow alterations, Eur. Heart J. Cardiovasc. Imag. 15 (12) (2014) 1400-1410.

[44] E. Nagel, T. Thouet, C. Klein, S. Schalla, A. Bornstedt, B. Schnackenburg, J. Hug, E. Wellnhofer, E. Fleck, Noninvasive determination of coronary blood flow velocity with cardiovascular magnetic resonance in patients after stent deployment, Circulation 107 (13) (2003) 1738-1743. 